\title{
Pomada de tomate contra quemaduras
}

\author{
Luz María Muñiz Noriega \\ 1mmuniz@cobaes.edu.mx \\ Colegio de Bachilleres del Estado de Sinaloa, Plantel 26
}

Eréndira Guadalupe Contreras Villagómez

erendira.contreras@cobaes.edu.mx

Egresada del Colegio de Bachilleres del Estado de Sinaloa, Plantel 26

Kristian Armando Pineda Castillo

kapineda@cobaes.edu.mx

Colegio de Bachilleres del Estado de Sinaloa, Plantel 60

Juan Carlos Angulo Ochoa

jcangulo@cobaes.edu.mx

Colegio de Bachilleres del Estado de Sinaloa, Plantel 27

México

\section{RESUMEN}

Se presentan avances de una investigación de corte experimental para el tratamiento de quemaduras leves con una pomada a base de tomate. El objetivo general fue demostrar que tan efectiva es la aplicación de una pomada elaborada artesanalmente con pulpa natural de tomate en quemaduras superficiales, con el propósito de aliviar o disminuir las molestias como el escozor, ardor o formación de ampollas originadas por este tipo de lesiones. Se siguió un enfoque cuantitativo para el análisis de los datos y pruebas. En lo que respecta al muestreo, un total de 16 participantes formaron parte del estudio. Los resultados obtenidos hasta el momento permitieron ver que, después de un tiempo de cinco minutos en promedio, el $100 \%$ de los individuos de prueba reportaron una disminución total de dolor con una sola aplicación de la pomada. Igualmente, no hubo presencia de ampollas de forma inmediata, aunque estas aparecieron posteriormente en un tiempo de entre 3 hasta 72 horas en el $37.5 \%$ de los casos; además, no hubo formación de ampollas en el restante $62.5 \%$ de los sujetos. En aquellos participantes que se formó ampolla, fue sin existencia de dolor o irritación.

Palabras clave: quemadura; jitomate; curación; lesión. 


\title{
Ointment against fleshburns
}

\begin{abstract}
Progress of an experimental investigation for the treatment of minor burns with a tomatobased ointment is presented. The general purpose was to demonstrate how effective is the application of an ointment made by hand with natural tomato pulp in superficial burns, in order to alleviate or reduce discomfort such as stinging, burning or blistering caused by this type of injury. A quantitative approach was followed for data analysis and tests. In terms of sampling, a total of 16 participants took part in the study. The results obtained so far showed that, after a time of five minutes on average, $100 \%$ of the test subjects reported a total decrease in pain with a single application of the ointment. Likewise, there was no immediate presence of blisters, although these appeared later in a time between 3 to 72 hours in $37.5 \%$ of the cases; furthermore, there was no blistering in the remaining $62.5 \%$ of the subjects. In those participants who did blister, it was without pain or irritation.
\end{abstract}

Keywords: burn; tomato; healing; injury.

Artículo recibido: 10 Agosto. 2021 Aceptado para publicación: 07. Setiembre. 2021 Correspondencia: 1mmuniz@ cobaes.edu.mx Conflictos de Interés: Ninguna que declarar 


\section{INTRODUCCIÓN}

Tradicionalmente, las plantas han sido utilizadas con fines curativos para diversas afecciones en el ser humano. A lo largo de la historia se han estudiado propiedades curativas de plantas; sus hojas, raíces, frutos, semillas, etc., las cuales combaten una diversa cantidad de enfermedades (Maldonado, Paniagua-Zambrana, Bussman, ZentenoRuiz y Fuentes, 2020). Además, existen diferentes formas farmacéuticas para transformar propiedades de plantas en tabletas, jarabes, tés, jabones, aceites, entre otros remedios. Una de las más utilizadas es la pomada, ya que resulta fácil de preparar, es económica, dura más tiempo y puede conservar las propiedades de floras eficazmente.

El tomate en específico tiene características particulares que lo hacen uno de los alimentos más ricos en propiedades tanto nutricionales como curativas. Dentro de las propiedades que se han encontrado resaltan las siguientes: aporta propiedades cicatrizantes, es refrescante e hidratante; adicionalmente, sirve para quemaduras puesto que sus componentes favorecen al equilibrio celular al regenerar la piel. Igualmente, un tomate perfectamente lavado puede eliminar cualquier posible bacteria en su superficie (Ballesteros y Pachón, 2017).

El presente trabajo gira entorno a los beneficios atribuidos al tomate, como auxiliar en el alivio de las molestias provocadas por quemaduras. Este es un problema muy común, el cual requiere de mayores alternativas de alivio inmediato, además de las acciones preventivas para evitar daños mayores. En este respecto, la investigación se enfoca al tratamiento de quemaduras leves aprovechando recursos disponibles para llevar a cabo las pruebas correspondientes.

Se espera que los resultados de esta investigación contribuyan a ofrecer un fundamento para profundizar en el estudio de las propiedades de este prodigioso alimento, pero que al mismo tiempo sirva para dar certeza en el uso tradicional que se le ha dado para aliviar quemaduras, un problema de nuestra cotidianidad y que requiere atención.

Uno de los problemas de salud que afectan mayormente a la población son los relacionados con quemaduras, que van desde lesiones leves hasta casos de quemaduras de segundo y tercer grado que comprometen la vida de personas por ser más severas.

En la actualidad, existen una gran diversidad de productos y remedios naturales que se utilizan como tratamientos de diversas molestias causadas por enfermedades o afecciones en el organismo. Según datos estadísticos de la Secretaría del Trabajo y Previsión Social 
(STPS, 2005-2017), hay miles de personas afectadas en accidentes de trabajo por quemaduras. De tal modo, es indiscutible la importancia de investigar diferentes alternativas para el tratamiento de quemaduras. Si bien es cierto, estas estadísticas se refieren a quemaduras en general, no se puede ignorar el hecho de que toda persona está en riesgo constante de sufrir estas lesiones, por lo que es necesario contar con tratamientos paliativos en hogares para tratar dichos incidentes.

Tradicionalmente, se ha usado el tomate para tratar este tipo de afecciones. No obstante, si se le agregan elementos que coadyuven a mejorar la eficacia del tratamiento, es decir, crear una forma farmacéutica como lo es una pomada, sobre la cual se explica más adelante, es probable que ayude mejor en la adsorción y distribución del principio activo del tomate en áreas cutáneas.

Según información que proporciona el sitio web Stanford Children’s Health (s.f.), las quemaduras de primer grado afectan únicamente la capa externa de la piel. Consecuentemente, el sitio de la quemadura se torna color rojizo, tiene la característica de ser doloroso, pero también seco y sin presencia de ampollas. En la mayoría de los casos, este tipo de lesiones son causadas por exposición excesiva al sol, o una explosión repentina y breve de calor. Las señales más comunes de una quemadura superficial incluyen el enrojecimiento en el área afectada, la piel seca, dolor al tacto, que generalmente dura de 48 a 72 horas, y desprendimiento de la piel.

\subsection{Beneficios y propiedades del tomate}

Según el sitio web Club Planeta (s.f) y expertos como Navarro-González y Periago, (2016), la planta de tomate, conocida científicamente como Solanum lycopersicum, popularmente llamada tomatera o jitomate, tiene dentro de su composición una importante cantidad de vitaminas $\mathrm{C}$ y E y sales minerales, las cuales le asignan muchos beneficios nutritivos. El tomate es un alimento que es muy versátil y es utilizado en casi todos los hogares en la preparación de alimentos. Se puede ingerir crudo, cocido o asado. Tiene grandes propiedades nutricionales que se han estudiado desde hace mucho tiempo; por ejemplo, se dice que este fruto tiene escasas calorías, la mayor parte de su peso es agua, además su sabor es ligeramente dulce debido a su contenido de hidratos de carbono y su sabor ácido se debe a algunos ácidos orgánicos.

Otras características del jitomate es que es fuente importante de ciertos minerales como el magnesio y potasio, un mineral esencial que ayuda a regular la presión sanguínea, 
contracciones musculares y reduce el riesgo de cálculos. Contiene además calcio, hierro, fósforo, triptófano y proteína. Es fuente de vitamina B1, B2, B5, E y la C. Presenta también carotenoides como el licopeno (pigmento que le da el color rojo característico al tomate), un antioxidante poderoso que puede ayudar a reducir el riesgo de algunos tipos de cáncer y enfermedades cardiovasculares. La vitamina $\mathrm{C}$ actúa también como un antioxidante. Puede ayudar a reducir la presión sanguínea y los niveles de colesterol; contribuye a la formación de tejido conjuntivo, mantiene la salud de los vasos capilares y de ese modo impide las contusiones leves y mantiene saludables las encías. Un jitomate de tamaño mediano suministra casi la mitad de la cantidad de vitamina $\mathrm{C}$ recomendada para el consumo diario, ayuda al desarrollo de una piel saludable, del cabello y de mucosas. Igualmente, sus efectos antioxidantes ayudan a lucir una piel más joven e hidratada y actúa como un limpiador facial natural, así como también favorece el aclarado de manchas, suaviza codos y rodillas (Servín, 2013).

Otro dato muy interesante es que una rodaja de jitomate sobre una quemadura leve proporciona alivio inmediato debido a la acción hidratante que reconstruye la elasticidad de la piel según la investigación realizada por Lepe (2015).

Por otro lado, el tomate tiene propiedades humectantes, además de que puede ser usado para problemas de la piel relacionados con quemaduras que no revistan demasiada gravedad. La razón por la que es posible utilizarlo para tratar quemaduras, se debe a sus propiedades cicatrizantes e hidratantes, que pueden beneficiar la sequedad de la piel que se genera en este tipo de lesiones (Esteban, s.f.).

\subsection{Problemática}

Uno de los mayores riesgos a la salud en la vida cotidiana y de mayor susceptibilidad sobre todo en menores de edad, son las quemaduras leves que suceden en hogares. Además, no existe una cultura de información o de difusión sobre tratamientos naturales, efectivos, de fácil acceso y baratos como lo es el tomate, que es uno de los productos más representativos y abundantes en México. Por lo tanto, existe un gran potencial en la elaboración y aplicación en el área de la salud de este prodigioso producto para tratar este tipo de lesiones cutáneas.

En tal, la investigación maneja la siguiente hipótesis: el tomate utilizado en forma de pomada es un auxiliar efectivo en el tratamiento de quemaduras leves para disminuir el dolor e irritación que se producen por tales heridas. 
Asimismo, el objetivo general del presente fue demostrar la efectividad de la pulpa natural de tomate en el tratamiento de quemaduras leves a través del uso de una pomada elaborada artesanalmente con el fin de aliviar o disminuir molestias causadas por este tipo de heridas, tales como el escozor o ardor y formación de ampollas. A manera de objetivos específicos, se planteó 1) elaborar de forma artesanal diferentes muestras de pomadas, realizando los ajustes y cálculos necesarios en diversas proporciones, en las cantidades de pulpa de tomate y demás ingredientes base, para lograr concentraciones diferentes y asegurar la efectividad del producto, y 2) probar en personas quemadas las diferentes muestras de pomada de tomate elaboradas, usando diferentes concentraciones de pulpa de tomate para lograr establecer la concentración exacta con mayor efectividad en el tratamiento para quemaduras leves.

\section{ESTRATEGIAS METODOLÓGICAS O MATERIALES Y MÉTODOS}

El enfoque de la investigación fue cuantitativo, siguiendo un diseño experimental "puro" (Hernández, Fernández y Baptista, 2014) ya que se elaboró una pomada de tomate que constó de varias etapas. Asimismo, se acompañó este diseño con una revisión documental con el objeto de adquirir información relacionada con las propiedades del tomate, tanto físicas como químicas, así como el procedimiento para la elaboración de pomadas.

Después de la revisión bibliográfica se llevó a cabo la fase de elaboración de la pomada. Para tal efecto, se consultó a un científico calificado quien facilitó a los autores del presente la formulación base de una pomada. Partiendo de esto, se realizaron diversas formulaciones con las variables del principio activo planteado en el presente. En esta fase se adquirieron los reactivos que consistieron en:

- Vaselina

- Lanolina

- Pulpa de tomate natural

- Vitamina E

- Benzoato de sodio

- Ácido salicílico

Los materiales o instrumentos necesarios para la elaboración fueron los siguientes:

- Balanza digital

- Vasos de precipitado de $50 \mathrm{ml}$

- Mortero con pistilo 
- Agitador de vidrio

- Espátula

- Cuchillo pequeño (para extraer la pulpa de tomate)

- Gotero

\subsection{Procedimiento}

Se elaboraron cinco muestras con concentraciones diferentes para realizar comparaciones y pruebas con el fin de medir la efectividad de cada una y elegir la concentración que sea más eficaz en el tratamiento de quemaduras leves, midiendo además el grado de alivio en los participantes de prueba de acuerdo con los parámetros que se tomaron en cuenta como enrojecimiento, escozor y dolor, además del tiempo que tardó en manifestarse el alivio esperado.

La elaboración de la pomada de tomate partió de una muestra de 100 gramos, para 20 gramos de producto, es decir, los cálculos se realizaron conforme a las proporciones que se dan a continuación:

\section{Muestra 1}

Las cantidades de reactivos fueron las siguientes: $10 \%$ de lanolina, $65 \%$ de vaselina y $25 \%$ de pulpa de tomate.

\section{Cálculos:}

\section{Lanolina:}

- $10 \mathrm{~g}$ lanolina $X\left(\frac{20}{100}\right)=2 g$ lanolina

- $65 \mathrm{~g}$ vaselina $X\left(\frac{20}{100}\right)=13 g$ vaselina

- $25 \mathrm{~g}$ pulpa de tomate $X\left(\frac{20}{100}\right)=5 g$ pulpa de tomate

\section{Muestra 2}

Se manejaron las cantidades de $20 \%$ de lanolina, $65 \%$ de vaselina y $15 \%$ de pulpa de tomate, una cápsula de vitamina $\mathrm{E}$ (antioxidante).

\section{Cálculos:}

- $20 \mathrm{~g}$ lanolina $X\left(\frac{20}{100}\right)=4 g$ lanolina

- $65 \mathrm{~g}$ vaselina $X\left(\frac{20}{100}\right)=13 g$ vaselina

- $15 \mathrm{~g}$ pulpa de tomate $X\left(\frac{20}{100}\right)=3 g$ de tomate

\section{Muestra 3}


Se manejaron las siguientes cantidades; $15 \%$ de lanolina, $64.45 \%$ de vaselina, $20 \%$ de pulpa de tomate, $0.05 \%$ de vitamina E (antioxidante), $0.5 \%$ de Benzoato de Sodio y $0.5 \%$ de ácido salicílico (conservadores).

\section{Cálculos:}

- $15 \mathrm{~g}$ lanolina $X\left(\frac{20}{100}\right)=3 g$ lanolina

- $65 \mathrm{~g}$ vaselina $X\left(\frac{20}{100}\right)=13 g$ vaselina

- $20 \mathrm{~g}$ pulpa de tomate $X\left(\frac{20}{100}\right)=4 g$ pulpa de tomate

\section{Cálculos de conservador:}

- $0.5 \mathrm{~g}$ Benzoato de sódio $X\left(\frac{20}{100}\right)=0.1 \mathrm{~g}$ benzoato de sodio

- $0.5 \mathrm{~g}$ ácido salicílico $X\left(\frac{20}{100}\right)=0.1 \mathrm{~g}$ ácido salicílico

\section{Cálculos de antioxidante:}

- 1 gota

\section{Muestra 4}

Se recurrió a las siguientes cantidades; $15 \%$ de lanolina, $63.95 \%$ de vaselina, $20 \%$ de pulpa de tomate, $0.05 \%$ de vitamina $\mathrm{E}$ (antioxidante), $0.5 \%$ de benzoato de sodio y $0.5 \%$ de ácido salicílico (conservadores).

\section{Cálculos:}

- $15 \mathrm{~g}$ lanolina $X\left(\frac{20}{100}\right)=3 g$ lanolina

- $63.95 \mathrm{~g}$ vaselina $X\left(\frac{20}{100}\right)=12.79 \mathrm{~g}$ vaselina

- $20 \mathrm{~g}$ pulpa de tomate $X\left(\frac{20}{100}\right)=4 g$ pulpa de tomate

\section{Cálculos de conservador:}

- $0.5 \mathrm{~g}$ benzoato de sodio $X\left(\frac{20}{100}\right)=0.1 \mathrm{~g}$ benzoato de sodio

- $0.5 \mathrm{~g}$ ácido salicílico $X\left(\frac{20}{100}\right)=0.1 \mathrm{~g}$ ácido salicílico

\section{Cálculos de antioxidante:}

- 0.05 Vitamina $\mathrm{E} X\left(\frac{20}{100}\right)=0.01 \mathrm{ml}$ vitamina $\mathrm{E}$

\section{Muestra 5:}


Las siguientes cantidades fueron manejadas; $10 \%$ de lanolina, 55\% de vaselina, $34.0 \%$ de pulpa de tomate, $0.05 \%$ de vitamina E (antioxidante), $0.5 \%$ de Benzoato de Sodio y $0.5 \%$ de ácido salicílico como (conservadores)

\section{Cálculos:}

- $10 \mathrm{~g}$ lanolina $X\left(\frac{20}{100}\right)=2 g$ lanolina

- $55 \mathrm{~g}$ vaselina $X\left(\frac{20}{100}\right)=11 \mathrm{~g}$ vaselina

- $34 \mathrm{~g}$ pulpa de tomate $X\left(\frac{20}{100}\right)=6.8 \mathrm{~g}$ pulpa de tomate

\section{Cálculos de conservador:}

- $0.5 \mathrm{~g}$ benzoato de sódio $X\left(\frac{20}{100}\right)=0.1 \mathrm{~g}$ benzoato de sodio

- $0.5 \mathrm{~g}$ ácido salicílico $X\left(\frac{20}{100}\right)=0.1 \mathrm{~g}$ ácido salicílico

\section{Cálculos de antioxidante:}

- 0.05 vitamina $\mathrm{E} X\left(\frac{20}{100}\right)=0.01 \mathrm{ml}$ vitamina $\mathrm{E}$

\subsubsection{Etapa de pruebas}

Cabe mencionar que la muestra número 1 , no tuvo vitamina $\mathrm{E}$, ni conservador, ni antioxidante. Además, se realizó con las proporciones descritas anteriormente y se guardó en refrigeración, logrando alcanzar en un primer periodo una conservación de 30 días, sin mostrar signos de enranciamiento o invasión microbiana.

A la muestra número 2 se le añadió el contenido de una cápsula de vitamina $\mathrm{E}$, y no se le añadieron conservadores, es decir, ni benzoato de sodio ni ácido salicílico. Al término de 48 horas, esta muestra presentó visible contaminación, al parecer fúngica presumiendo que se debió al exceso de vitamina $\mathrm{E}$, dado que en la muestra anterior que no tenía este reactivo no se observó crecimiento de microorganismos de apariencia (sin confirmar) fúngica. Evidentemente, esta muestra no fue probada.

Las muestras 3 y 4 fueron probadas y no han presentado signos de contaminación, además están en observación para determinar la caducidad de estas.

La muestra 5 contuvo una concentración diferente, presentando una disminución de la cantidad de vaselina y un aumento en el porcentaje de pulpa de tomate. Las concentraciones fueron las siguientes: $10 \%$ de lanolina, 55\% de vaselina, $34.0 \%$ de pulpa de tomate con respecto a la muestra 4 que está como sigue: $15 \%$ de lanolina, $64.45 \%$ de vaselina, $20 \%$ de pulpa de tomate. Se preparó esta última muestra para determinar el 
periodo de conservación del producto tanto en condiciones ambientales como en refrigeración.

El producto resultante se colocó en un envase adecuado. Para tal efecto, se diseñó en una primera etapa un boceto o dibujo para representar la pomada y contar con un prototipo atractivo para consumidores, además de elaborar presentaciones pequeñas para mayor practicidad (Figura 1).

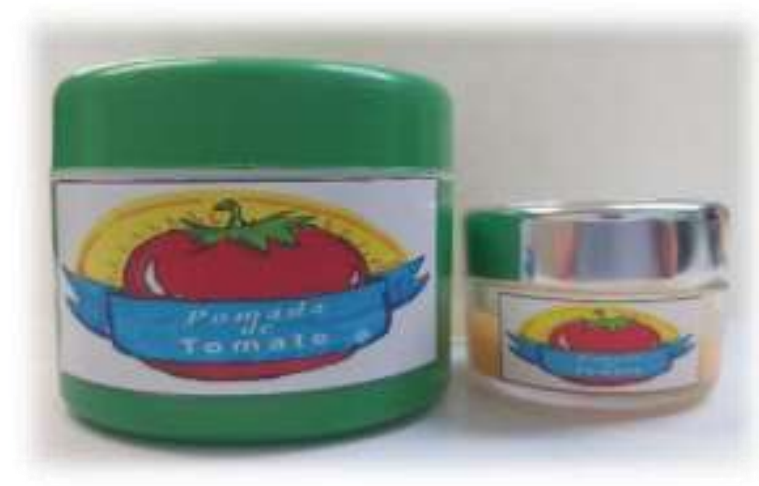

Figura 1. Presentación de envase para la pomada de tomate.

\section{Muestreo}

Se contó con un total de 16 participantes, a quienes en la etapa de pruebas se les aplicaron las muestras tres y cuatro a 14 de ellos, jóvenes estudiantes voluntarios de primer grado de bachillerato, así como dos adultos. El procedimiento fue el siguiente:

Se expuso a los voluntarios a una fuente de calor, para ello, se calentó a fuego directo una lámina de hierro y se puso en contacto por un instante con la mano izquierda en la parte dorsal, en la base del pulgar. Esto para asemejar o simular condiciones bajo las cuales ocurren los accidentes en el hogar al sufrir quemaduras de este tipo. Cabe destacar que la exposición a la fuente de calor no puso en riesgo la salud de los participantes, quienes fueron informados oportunamente de las pruebas a las que serían sometidos y sus riesgos; los padres de los menores consintieron la participación de los adolescentes voluntarios (Figura 2). 


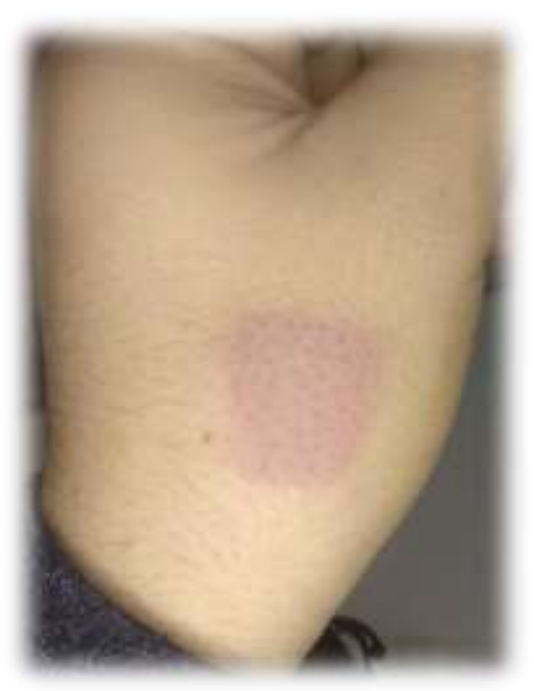

Figura 2. Quemadura de primer grado, provocada intencionalmente para el experimento.

Después del contacto y sufrir la quemadura provocada, se colocó inmediatamente una porción generosa de pomada en la parte afectada dando masaje hasta su absorción (Figura 3).

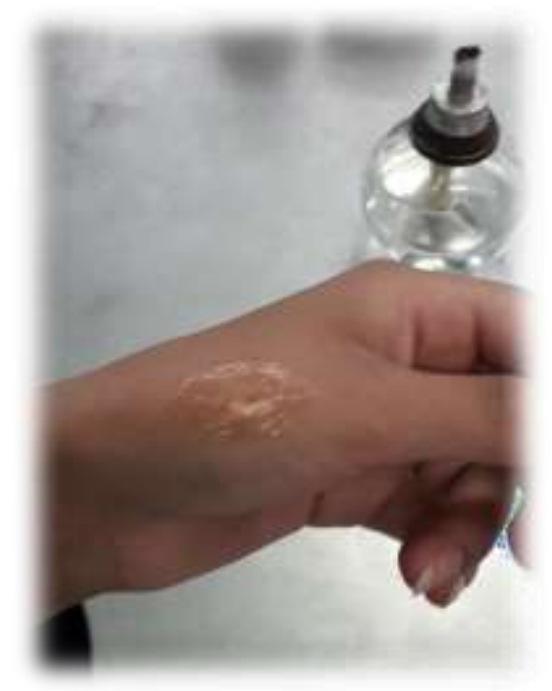

Figura 3. Aplicación de la pomada de tomate.

Por otra parte, se subraya el hecho de que fue necesario crear las condiciones de exposición a quemaduras ya que resultaba poco práctico colocar el producto en los hogares y esperar a que sucedieran accidentes de este tipo para realizar las pruebas, razón por la cual fue necesario establecer o simular condiciones para probar la eficacia de la pomada. 


\section{RESULTADOS Y DISCUSIÓN}

Después de un espacio de tiempo de cinco minutos en promedio, el 100\% de los sujetos de prueba manifestaron una disminución total de dolor. Además, no hubo formación de ampollas de forma inmediata, aunque estas aparecieron en el $37.5 \%$ de los casos en un periodo posterior a la aplicación de la pomada en un periodo de tiempo de entre 3 hasta 72 horas. Por otro lado, en el resto, es decir el $62.5 \%$ no hubo aparición de ampollas. Es fundamental recalcar que la formación de ampolla fue sin existencia de dolor o irritación. Igualmente, es digno de mencionar que solo se realizó una sola aplicación de la pomada para el tratamiento de quemaduras.

Es importante mencionar que la indagación de la investigación sigue en curso. Aunque los hallazgos presentan avances significativos en cuanto a la hipótesis planteada, se reconoce que es fundamental realizar más pruebas y continuar con observaciones entorno a los participantes con el afán de dar seguimiento a la mejora o alivio de las lesiones ocasionadas por las quemaduras provocadas intencionalmente para efectos del estudio. Por tal, se recomienda ampliamente a los lectores esperar a que se realicen más pruebas relacionadas con el presente, además de poner siempre en consideración la opinión de un médico para el tratamiento de heridas por quemaduras y jamás automedicarse.

\section{CONSIDERACIONES FINALES}

En base a los resultados obtenidos y considerando que esta investigación aún continúa, se puede destacar que el conocimiento empírico que se maneja de forma tradicional tiene fundamentos científicos y procedimentales válidos, ya que los datos hasta hoy obtenidos arrojan elementos suficientes para confirmar la hipótesis planteada. De este modo, se puede apreciar la manera en que se alcanzó el objetivo general, al lograr demostrar hasta cierto punto la efectividad de la pomada de tomate para aliviar y combatir molestias causadas por quemaduras de primer grado. Por lo tanto, se considera que se sientan bases para la formulación de una teoría sólida que apoya el poder curativo del tomate en este tipo de afecciones de la piel.

Adicionalmente, se reitera que este prodigioso alimento tiene grandes propiedades tanto alimentarias como medicinales, pero siempre debe estar como primera opción la consulta a un médico antes de iniciar cualquier tipo de tratamiento curativo. Se considera que se debe profundizar en la investigación para obtener un producto que se encuentre al alcance de los hogares ya que las quemaduras representan, como ya se analizó en este trabajo, un 
porcentaje importante de problemas de salud en la población. Finalmente, se espera que se aprovechen los conocimientos generados en esta investigación y que se pueda generar un beneficio a la población.

Es fundamental subrayar que la investigación no está concluida. Los hallazgos que se presentan son solo un avance en las pruebas realizadas. Por tal motivo, el uso que se le dé a la información del presente artículo queda bajo responsabilidad de los lectores, por lo que los autores se deslindan de cualquier responsabilidad o perjuicio que se desprenda del uso de esta información que pretende ser meramente para usos educativos e informativos.

\section{LISTA DE REFERENCIAS BIBLIOGRAFICAS}

Ballesteros, M. C., \& Pachón, J. D. (2017). Desarrollo de un producto derivado del tomate y su proceso de transformación en la finca productora "El Regalito" de Fómeque, Cundinamarca (tesis de pregrado) Universidad de La Salle, Bogotá D.C. Colombia. Recuperado de https://ciencia.lasalle.edu.co/cgi/viewcontent.cgi?article=1036\&context=ing _industrial

Club planeta. (s.f.). Jitomate propiedades y beneficios a la salud. Recuperado de https://www.clubplaneta.com.mx/cocina/jitomate_propiedades_y_beneficios _a_la_salud.htm

Esteban, J. (s.f.), Tomate para las quemaduras. Recuperado de https://www.plantasparacurar.com/tomate-para-las-quemaduras/

Hernández, R., Fernández, C., \& Baptista, P. (2014). Metodología de la investigación (6a. ed.). McGraw-Hill.

Lepe, N. (06 de enero del 2015). Derribando mitos cuanto sirve usar rodajas de tomate para tratar la insolación. Recuperado de

https://www.publimetro.cl/cl/nacional/2015/01/06/derribando-mitos-cuantosirve-rodajas-tomate-tratar-insolacion.html

Maldonado, C., Paniagua-Zambrana, Bussman, N., Zenteno-Ruiz, R. W., \& Fuentes, A. F. (2020). La importancia de las plantas medicinales, su taxonomía y la búsqueda de la cura a la enfermedad que causa el coronavirus (COVID-19). Ecología en Bolivia, 55(1), 1-5. Recuperado de http://www.scielo.org.bo/scielo.php?script=sci_arttext\&pid=S1605- 
25282020000100001

Navarro-González, I. \& Periago, M. J. (2016). El tomate, ¿alimento saludable y/o funcional? Revista Española de Nutrición Humana y Dietética, 20(4), 323335. https://dx.doi.org/10.14306/renhyd.20.4.208

Secretaría del Trabajo y Previsión Social. (2005-2017). Información sobre Accidentes y Enfermedades de Trabajo Nacional 2005-2017. Secretaría del Trabajo y Previsión Social. Recuperado de https://autogestionsst.stps.gob.mx/Proyecto/Content/pdf/Estadisticas/Nacion al.pdf

Servín, M. del C. (2013). Nutrición básica y aplicada. México, México: Universidad Nacional Autónoma de México. Recuperado de http://www.eneo.unam.mx/publicaciones/publicaciones/ENEO-UNAMNutricionBasicayAplicada.pdf

Stanford Children's Health. (s.f.). Quemaduras de primer grado (superficiales). Recuperado de https://www.stanfordchildrens.org/es/topic/default?id=quemadurasdeprimerg rado-90-P04847 